\title{
Study of the Impact of Tissue Density Heterogeneities on 3-Dimensional Abdominal Dosimetry: Comparison Between Dose Kernel Convolution and Direct Monte Carlo Methods
}

\author{
Arnaud Dieudonné*1,2, Robert F. Hobbs*3 ${ }^{* 3}$, Rachida Lebtahi ${ }^{1,2,4}$, Fabien Maurel ${ }^{5}$, Sébastien Baechler ${ }^{6}$, Richard L. Wahl ${ }^{3}$, \\ Ariane Boubaker ${ }^{7}$, Dominique Le Guludec ${ }^{1,4}$, Georges Sgouros ${ }^{3}$, and Isabelle Gardin ${ }^{8,9}$ \\ ${ }^{I}$ Department of Nuclear Medicine, Beaujon Hospital, Assistance Publique-Hôpitaux de Paris (APHP), Clichy, France; ${ }^{2}$ INSERM \\ U773, Paris, France; ${ }^{3}$ Department of Radiology and Radiological Science, Johns Hopkins University, School of Medicine, Baltimore, \\ Maryland; ${ }^{4}$ University Paris Diderot-Paris 7, Paris, France; ${ }^{5}$ Department of Nuclear Medicine, Nice University Hospital, Nice, \\ France; ${ }^{6}$ Institute of Radiation Physics, Lausanne University Hospital, Lausanne, Switzerland; ${ }^{7}$ Nuclear Medicine Department, \\ Lausanne University Hospital, Lausanne, Switzerland; ${ }^{8}$ LITIS (EA 4108), University of Rouen, Rouen, France; and ${ }^{9}$ Department of \\ Nuclear Medicine, Henri Becquerel Center and CHU, Rouen, France
}

Dose kernel convolution (DK) methods have been proposed to speed up absorbed dose calculations in molecular radionuclide therapy. Our aim was to evaluate the impact of tissue density heterogeneities (TDH) on dosimetry when using a DK method and to propose a simple density-correction method. Methods: This study has been conducted on 3 clinical cases: case 1, nonHodgkin lymphoma treated with ${ }^{131}$ I-tositumomab; case 2, a neuroendocrine tumor treatment simulated with ${ }^{177} \mathrm{Lu}$-peptides; and case 3 , hepatocellular carcinoma treated with ${ }^{90}$ Y-microspheres. Absorbed dose calculations were performed using a direct Monte Carlo approach accounting for TDH (3D-RD), and a DK approach (VoxelDose, or VD). For each individual voxel, the VD absorbed dose, $\mathrm{D}_{\mathrm{VD}}$, calculated assuming uniform density, was corrected for density, giving $D_{\text {VDd }}$. The average 3D-RD absorbed dose values, $\mathrm{D}_{3 \mathrm{DRD}}$, were compared with $\mathrm{D}_{\mathrm{VD}}$ and $\mathrm{D}_{\mathrm{VDd}}$, using the relative difference $\Delta_{\mathrm{VD} / 3 \mathrm{DRD}}$. At the voxel level, density-binned $\Delta_{\mathrm{VD} / 3 \mathrm{DRD}}$ and $\Delta_{\mathrm{VDd} / 3 \mathrm{DRD}}$ were plotted against $\rho$ and fitted with a linear regression. Results: The $D_{V D}$ calculations showed a good agreement with $\mathrm{D}_{3 \mathrm{DRD}} . \Delta_{\mathrm{VD} / 3 \mathrm{DRD}}$ was less than $3.5 \%$, except for the tumor of case $1(5.9 \%)$ and the renal cortex of case 2 (5.6\%). At the voxel level, the $\Delta_{\mathrm{VD} / 3 \mathrm{DRD}}$ range was $0 \%-14 \%$ for cases 1 and 2 , and $-3 \%$ to $7 \%$ for case 3 . All 3 cases showed a linear relationship between voxel bin-averaged $\Delta_{\mathrm{VD} / 3 \mathrm{DRD}}$ and density, $\rho$ : case $1(\Delta=-0.56 \rho+0.62$, $\left.R^{2}=0.93\right)$, case $2\left(\Delta=-0.91 \rho+0.96, R^{2}=0.99\right)$, and case $3\left(\Delta=-0.69 \rho+0.72, R^{2}=0.91\right)$. The density correction improved the agreement of the DK method with the Monte Carlo approach $\left(\Delta_{\mathrm{VDd} / 3 \mathrm{DRD}}<1.1 \%\right)$, but with a lesser extent for the tumor of case $1(3.1 \%)$. At the voxel level, the $\Delta_{\mathrm{VDd} / 3 \mathrm{DRD}}$ range decreased for the 3 clinical cases (case 1, $-1 \%$ to $4 \%$; case 2 , $-0.5 \%$ to $1.5 \%$, and $-1.5 \%$ to $2 \%$ ). No more linear regression existed for cases 2 and 3, contrary to case $1(\Delta=0.41 \rho-0.38$, $\left.R^{2}=0.88\right)$ although the slope in case 1 was less pronounced.

Received Mar. 19, 2012; revision accepted Aug. 29, 2012.

For correspondence or reprints contact: Arnaud Dieudonné, Service de Médecine Nucléaire, Hôpital Beaujon, 100, Boulevard du Général Leclerc, 92110 Clichy, France.

E-mail: arnaud.dieudonne@bjn.aphp.fr

${ }^{*}$ Contributed equally to this work.

Published online Dec. 18, 2012

COPYRIGHT @ 2013 by the Society of Nuclear Medicine and Molecular Imaging, Inc.
Conclusion: This study shows a small influence of TDH in the abdominal region for 3 representative clinical cases. A simple density-correction method was proposed and improved the comparison in the absorbed dose calculations when using our voxel $\mathrm{S}$ value implementation.

Key Words: 3D dosimetry; tissue density; convolution; Monte Carlo

J Nucl Med 2013; 54:236-243

DOI: 10.2967/jnumed.112.105825

$\mathbf{M}$ olecular radionuclide therapy with ${ }^{131}$ I has been the standard treatment for thyroid carcinoma for more than $50 \mathrm{y}$ of clinical practice, but its treatment planning remains based on a standard amount of activity defined according to the nature of the residual tissues (1). Other targeted radionuclide therapy (TRT) modalities have been developed such as peptide receptor radionuclide therapy for neuroendocrine tumors (NET), radioimmunotherapy for antibodytargeted tumors (e.g., non-Hodgkin lymphomas [NHL]), and selective internal radiation therapy for hypervascularized tumors, all with the potential for toxicity to normal tissues. At this time, only the treatment regimen of NHL with ${ }^{131}$ I-tositumomab requires patient-specific dosimetry. Indeed, whole-body dosimetry is performed using a dosimetric dose of ${ }^{131}$ I-tositumomab before the patient therapeutic administration (2). However, most of the aforementioned TRT modalities are still administered using a standard amount of radioactivity or at best by adapting the amount of radioactivity to the patient body weight or surface area $(3,4)$.

Individual dosimetry can be implemented with a certain level of personalization using 3-dimensional (3D) quantification and standard absorbed dose coefficients ( $\mathrm{S}$ value), which are available from official MIRD publications (5-7) and can be corrected for patient organ mass (8). Indeed, 3D quantification methods using SPECT or PET can be implemented 
in clinical routine (9), using full iterative reconstruction, including CT-based attenuation correction, as well as scatter effect and collimator response compensations. The software package OLINDA/EXM (10) provides the tools for organ and suborgan dosimetry, including biologic data-fitting and a comprehensive $S$ value library. However, several limitations have already been pointed out, mainly regarding the impossibility of accounting for heterogeneous activity distribution within compartments (11) and the simplicity of the spheric tumor model and tumor inclusion (12).

A much higher level of personalization can be obtained with a fully $3 \mathrm{D}$ dosimetry for which each step of the dosimetry is performed at the voxel level to allow editing of descriptive statistics, dose-volume histograms (DVHs), and isodose curves.

At this level of personalization, 2 options are available: direct Monte Carlo simulation (MC) and dose kernel convolution (DK).

Direct MC methods are based on generic MC codes such as MCNP (Monte Carlo N-Particle) or EGS (Electron $\gamma$-Shower). They can take into account both radionuclide and tissue density distributions, thus presumably leading to an accurate absorbed dose distribution assessment. For these reasons, MC methods are considered as the reference. MC methods have been implemented in software tools, such as 3D-RD (13), SIMDOS (14), and OEDIPE (15).

To speed up the computation, DK approaches have also been proposed. The first was the dose point-kernel method (16), which was later extended to voxel sources (dose voxel-kernel) in MIRD pamphlet 17 (17) by the introduction of voxel $\mathrm{S}$ values (VSV). Absorbed dose calculation by DK, either by dose point-kernel or dose voxel-kernel, can take into account radionuclide distribution at the voxel level, but rapid implementations using fast Fourier transform or fast Hartley transform impose the use of an invariant kernel and, thus, the assumption of uniform tissue density. The DK methods are implemented in software tools such as VoxelDose (18) and RMDP (19).

Direct MC simulations involve a higher computation time than convolution approaches, particularly if one wants to achieve a low statistical noise at the voxel level, making it challenging for clinical practice. On the other hand, fast implementations of DK approaches may be not suitable for highly heterogeneous body regions such as the thorax or bone sites. It has been shown that the differences between the 2 approaches in a homogeneous medium amount to no more than small discrepancies in the average absorbed dose and at the voxel level $(11,20)$. Consequently, the DK approaches, in cases for which the homogeneous tissue density assumption can be made, are equivalent to direct MC computations.

Previous studies have discussed the impact of tissue heterogeneities on the energy deposition of electrons, especially on the backscatter effect (21-24). Indeed, the perturbations of material interfaces were quantified in terms of backscatter dose factor as described by Buffa et al. (23) for point sources. The feasibility of using dose point-kernel scaling methods has been shown by Janicki et al. (25) and Loudos et al. (26). Furthermore, Furhang et al. (27) have included an equivalent distance method in an MC approach.

The dosimetry of TRT is often focused in the abdomen either for tumor localization (NETs, hepatic hypervascularized tumors, or NHLs) or for organs at risk (liver, kidney, or spleen). Indeed, in radioimmunotherapy, tumors can be present in the abdominal region and toxicity can occur for liver, kidneys, or spleen (28). In peptide receptor radionuclide therapy, tumors can be present in the abdomen, and the kidney is an organ at risk because of the retention of radiopeptides or radiometals (29). Also, the goal of selective internal radiation therapy is to control the tumor while preserving the nontumoral liver (30).

Consequently, our goal was to study the influence of tissue density heterogeneities (TDH) on abdominal 3D dosimetry and in extend, the hypothesis of a homogeneous tissue density. The use of simple density correction methods at the voxel and organ level, similar to the organ mass correction proposed in MIRD pamphlet $5(8)$, was also investigated. To this aim, 3 representative clinical cases were studied.

\section{MATERIALS AND METHODS}

\section{Input Data}

The 3 clinical cases included the following: case 1, a NHL patient treated with unlabeled tositumomab, followed by ${ }^{131}{ }^{1}$ I-tositumomab (Bexxar therapeutic regimen; GlaxoSmithKline); case 2, a patient who had clinically suspected NETs and for whom dosimetry for possible therapy using ${ }^{177} \mathrm{Lu}$-peptides was simulated; and case 3, a hepatocellular carcinoma (HCC) patient treated with ${ }^{90}$ Y-loaded microspheres.

For each case, the input data were the time-integrated activity (TIA) map derived from the SPECT images and the density map obtained from the CT scan and interpolated to the SPECT voxel size. Both dose calculations (MC and DK) were based on the $\beta^{-}$, atomic electron, and photon components of ${ }^{131} \mathrm{I},{ }^{177} \mathrm{Lu}$, and ${ }^{90} \mathrm{Y}$ decay spectra obtained from the RADTABS program (31).

\section{D-RD}

The MC calculation was performed with the software tool 3D-RD (32), based on the EGSnrc (EGS National Research Council Canada) code. A more complete description of 3D-RD has been previously provided (13). Briefly, 10 million events were run using 8 parallel processes on a node of a Beowulf-class cluster (EGSnrc MC software). The output values from the MC method given as energy deposition distribution from the contributing components per decaying particle were weighted for probability and TIA and then converted to absorbed dose for each voxel by dividing by the voxel mass. The absorbed dose $\mathrm{D}_{3 \mathrm{DRD}}$ for each volume of interest (VOI) as a whole was calculated by summing the energy depositions and masses in the VOIs separately and then dividing the VOI energy by the VOI mass. To take into account tissue heterogeneity, tissue density derived from the patient's CT data was integrated in the MC calculation.

\section{VoxelDose}

The DK calculation, implemented using VSV, was performed with the software tool VoxelDose (18). This implementation will be referred to as VD. The VSVs were precalculated with MC code MCNPX (33) at a fine voxel size, with the tissue medium set to 
soft tissue as defined by the International Commission on Radiation Units and Measurements $\left(\rho=1.04 \mathrm{~g} \cdot \mathrm{cm}^{-3}\right)(34)$. The dose calculation itself, as described by Dieudonné et al. (11), encompasses the resampling of the VSV at the voxel size of the TIA map and the rapid convolution via a fast Hartley transform, giving the absorbed dose $\mathrm{D}_{\mathrm{VD}}$ for each voxel of the TIA map.

\section{Density Correction}

To account for heterogeneous density distribution, a voxel density correction was applied to correct the absorbed dose within each voxel $\mathrm{D}_{\mathrm{VD}}(x, y, z)$ with the voxel densities $\rho(x, y, z)$ to obtain $\mathrm{D}_{\mathrm{VDd}}(x, y, z)$, using Equation 1. This implementation will be referred to as VDd.

$$
\mathrm{D}_{\mathrm{VDd}}(x, y, z)=\mathrm{D}_{\mathrm{VD}}(x, y, z) \times \frac{1.04}{\rho(x, y, z)} .
$$

According to MIRD pamphlet 5 (8), an average organ density correction was also applied to the average absorbed dose of each target $r_{\mathrm{T}}$, to compensate for the differences between a uniform density of $1.04 \mathrm{~g} \cdot \mathrm{cm}^{-3}$ and real density measurement from a voxelized density map $\rho(x, y, z)$, following Equation 2. This implementation will be referred to as VD̄. This correction applies to the electron (atomic and $\beta^{-}$) and photon decay components and is correct only for the local energy deposition of secondary electrons.

$$
\mathrm{D}_{\mathrm{VD \overline {d }}}\left(\mathrm{r}_{\mathrm{T}}\right)=\mathrm{D}_{\mathrm{VD}}\left(\mathrm{r}_{\mathrm{T}}\right) \times \frac{1.04}{\rho\left(\mathrm{r}_{\mathrm{T}}\right)} .
$$

\section{Absorbed Dose Comparison}

The absorbed dose maps computed with 3D-RD were used as the reference. The comparison between the 3 VSV implementations (VD, VDd, and VD $\bar{d}$ ) was performed in terms of absorbed dose relative differences $\left(\Delta_{\mathrm{VD} / 3 \mathrm{DRD}}=\frac{\left.\mathrm{D}_{\mathrm{VD}}-\mathrm{D}_{3 \mathrm{DRD}}\right)}{\mathrm{D}_{3 \mathrm{DRD}}}\right)$ at the organ-tumor level and voxel level and in terms of DVH.

At the voxel level, density-binned $\mathrm{D}_{\mathrm{VD} / 3 \mathrm{DRD}}$ and $\Delta_{\mathrm{VDd} / 3 \mathrm{DRD}}$ were plotted against the density $\rho$ within VOIs and fitted with a linear regression for absorbed doses above $1 \mathrm{~Gy}$ and density values above $0.90 \mathrm{~g} \cdot \mathrm{cm}^{-3}\left(0.96 \mathrm{~g} \cdot \mathrm{cm}^{-3}\right.$ for case 3 , for which the density values were less dispersed). The analyses included $95.1 \%$ of the voxels in the organs at risk (kidneys, liver, and spleen) for the radiopeptide case, corresponding to $98.1 \%$ of the absorbed dose to those regions. For the lymphoma case, $95.1 \%$ of the tumor voxels were used, corresponding to $95.8 \%$ of the dose in the tumor. For the HCC case, $57.7 \%$ of the voxels in the liver were used, corresponding to $99.0 \%$ of the dose. The discrepancy between these last 2 numbers is due in large part to the fact that only the right lobe of the liver was treated and due to localization of the activity uptake in the treated lobe.

The DVHs were computed in the tumor of case 1 and in tumor and healthy liver for case 3 .

Case 1: ${ }^{131}$ I-Tositumomab. Case 1 concerns a NHL patient with a periaortal abdominal tumor who was imaged after the administration of $200 \mathrm{MBq}$ of ${ }^{131}$ I-tositumomab as a tracer and subsequently treated with the same radiopharmaceutical. This patient's data have previously been described and published (35). Abdominal SPECT/CT images were acquired on a Phillips Precedence SPECT/CT scanner (16-slice) at 48, 72, and $144 \mathrm{~h}$ after tracer administration. The SPECT images were reconstructed using the QSPECT method (30 iterations, 16 subsets per iteration) $(36,37)$, based on the iterative orderedsubsets expectation maximization algorithm with reconstruction-based compensation for attenuation, scatter, and the collimator-detector response function. The resulting matrix was $128 \times 128 \times 87(3.91 \times$ $3.91 \times 4.25 \mathrm{~mm})$. The different reconstructed SPECT images were then registered over time using a HERMES workstation. The activities for the different voxels were fitted as a function of time with a hybrid trapezoid-exponential fit and integrated to obtain the voxel TIAs. The defined VOIs for this patient were the kidneys, spleen, liver, and tumor.

Case 2: ${ }^{177}$ Lu-Peptide. Case 2 concerns a patient with clinically suspected NETs, examined at the University Hospital of Lausanne and injected with $185 \mathrm{MBq}$ of ${ }^{111}$ In-pentetreotide (OctreoScan; Covidien) for diagnostic imaging. Three SPECT images of the abdominal region were acquired at 4, 24, and $48 \mathrm{~h}$ after injection with a 3-head $\gamma$-camera (Triad; Trionix Research Laboratory, Inc.), using a medium-energy collimator and $15 \%$ energy windows centered at 171 and $245 \mathrm{keV}$. This patient's data have also been described and published previously (38). The SPECT images were reconstructed and registered in a manner identical to that in case 1 . The resulting matrix was $128 \times 128 \times 128(4.48 \times 4.48 \times 4.48 \mathrm{~mm})$. The voxelized activity was adjusted for the difference in physical halflife between the pretherapeutic $\left({ }^{111} \mathrm{In}, 67.3 \mathrm{~h}\right)$ and planned therapeutic $\left({ }^{177} \mathrm{Lu}, 6.73 \mathrm{~d}\right)$ isotopes. The functional fit to the voxelized activities was a simple exponential. VOI contours were drawn for the kidneys, liver, and spleen. Furthermore, a distinction was made between the renal cortices and medullae. This patient was not treated because no tumors were evidenced in the ${ }^{111}$ In images. This case was selected for its relevance to ${ }^{177} \mathrm{Lu}$ renal dosimetry.

Case 3: ${ }^{90} Y$-Microspheres. Case 3 concerns an HCC treated with $1.0 \mathrm{GBq}$ of ${ }^{90}$ Y-loaded resin microspheres. The data were collected from the pretherapeutic procedure, which requires the injection of ${ }^{99 \mathrm{~m}} \mathrm{Tc}$-macroaggregated albumin (MAA) (30), that is, ${ }^{99 \mathrm{~m}} \mathrm{Tc}$ injected activity and a ${ }^{99 \mathrm{~m}} \mathrm{Tc}-\mathrm{MAA}$ SPECT/CT dataset. The patient had a perfused volume of ${ }^{99 \mathrm{~m}} \mathrm{Tc}-\mathrm{MAA}$ of $1,500 \mathrm{~cm}^{3}$ and a liver tumor of $714 \mathrm{~cm}^{3}$. The ${ }^{99 \mathrm{~m} T c-M A A ~ S P E C T / C T ~ d a t a s e t ~}$ showed a potential ${ }^{90} \mathrm{Y}$ gastric uptake. The SPECT/CT data were acquired on a Symbia T2 $\gamma$-camera (Siemens Healthcare) at the nuclear medicine department of Beaujon Hospital. The data processing has been described and published previously (39). The reconstruction was performed using a 3D ordered-subsets expectation maximization algorithm (6 iterations and 8 subsets) on Syngo MI workplace (MIWP; Siemens Healthcare) with attenuation, scatter, and nonuniform collimator response compensations. The reconstructed SPECT voxel size was $4.8 \times 4.8 \times 4.8 \mathrm{~mm}$. The CT image was registered with MIWP. The TIA map was derived from the ${ }^{99 \mathrm{~m}}$ Tc-MAA SPECT/CT dataset, assuming similar distributions within the liver for ${ }^{90} \mathrm{Y}$-microspheres and ${ }^{99 \mathrm{~m}} \mathrm{Tc}-\mathrm{MAA}$ after adjustment was made for the difference in radionuclide physical half-life (64.1 vs. $6.02 \mathrm{~h}$, respectively).

This study was approved by the local ethical committee, and all patients signed a written informed consent form.

\section{RESULTS}

With the MC calculation, 2-3 h were required to generate 10 million events using a single cluster node (with 8 parallel processes), for each time point and decay type (photon, $\beta$-particle, and atomic electron). On a desktop computer, the calculation time for the DK method was below $10 \mathrm{~s}$ for each case.

The comparison of the average absorbed dose to organs and tumors is given for each case in Tables 1-3. Only 2 absorbed dose differences between $D_{3 D R D}$ and $D_{V D}$ 


\begin{tabular}{|c|c|c|c|c|c|c|c|c|c|c|}
\hline \multirow[b]{3}{*}{ Structure of interest } & \multirow[b]{3}{*}{ TIA (MBq.s) } & \multirow[b]{3}{*}{ Mass (g, with $\mathrm{d}(x, y, z))$} & \multirow[b]{3}{*}{ Mass $(\mathrm{g}$, with $\mathrm{d}=1.04)$} & \multicolumn{7}{|c|}{ Average absorbed dose (Gy) and RD (\%) } \\
\hline & & & & \multirow[b]{2}{*}{ 3D-RD } & \multicolumn{2}{|c|}{ VD } & \multicolumn{2}{|c|}{ VDd } & \multicolumn{2}{|c|}{ VD̄ } \\
\hline & & & & & Gy & $\%$ & Gy & $\%$ & Gy & $\%$ \\
\hline Tumor & 165 & 693 & 706 & 10.4 & 9.77 & -5.9 & 10.0 & -3.1 & 9.95 & -3.6 \\
\hline Liver (partial) & 22.9 & 815 & 804 & 1.59 & 1.59 & 0.0 & 1.59 & -0.06 & 1.57 & -1.3 \\
\hline Spleen (partial) & 6.52 & 173 & 172 & 1.87 & 1.86 & -0.5 & 1.87 & 0.21 & 1.85 & -1.2 \\
\hline Kidneys & 46.3 & 594 & 612 & 3.86 & 3.73 & -3.5 & 3.88 & -0.52 & 3.84 & -0.42 \\
\hline
\end{tabular}

$\left(\Delta_{\mathrm{VD} / 3 \mathrm{DRD}}\right)$ exceeded $5 \%$. The highest difference from the 3D-RD result was found for the tumor in case 1 ( ${ }^{131}$ I-tositumomab treatment), for which VD reported a lower absorbed dose by $-5.9 \%$. The second value was found in case $2\left({ }^{177} \mathrm{Lu}\right.$-peptide treatment) for the renal cortex dosimetry $(5.6 \%)$. Otherwise, the differences were less than $3.5 \%$ for all organs and tumors within the 3 cases.

Transverse slices of the 3D absorbed dose maps computed with 3D-RD and VoxelDose are given in Figure 1 for each case. At the voxel level, the $\Delta_{\mathrm{VD} / 3 \mathrm{DRD}}$ ranged from $0 \%$ to $14 \%$ for cases 1 and 2 and from $-3 \%$ to $7 \%$ for case 3 . In Figures $2 \mathrm{~A}, 2 \mathrm{C}$, and $2 \mathrm{E}$, the density-binned $\Delta_{\mathrm{VD} / 3 \mathrm{DRD}}$ plotted against the density $\rho$ for absorbed doses above 1 Gy are presented. A linear relationship was found, showing that the absorbed dose differences are strongly related to $\rho$ for each case: case 1: $\Delta_{\mathrm{VD} / 3 \mathrm{DRD}}(\%)=-55.6 \rho+61.9, R^{2}=0.93$; case $2: \Delta_{\mathrm{VD} / 3 \mathrm{DRD}}(\%)=-91.2 \rho+96.1, R^{2}=0.99$; and case 3: $\Delta_{\mathrm{VD} / 3 \mathrm{DRD}}(\%)=-69.3 \rho+71.8, R^{2}=0.91$.

The use of density correction on VD computation globally improved the agreement with 3D-RD. At the organ and tissue levels, both VDd and VDd gave comparable results. Values of $\Delta_{\mathrm{VDd} / 3 \mathrm{DRD}}$ and $\Delta_{\mathrm{VDd} / 3 \mathrm{DRD}}$ were less than $2 \%$, except for the tumor in case 1 , for which relative differences of $-3.6 \%$ and $3.1 \%$ were found, respectively. At the voxel level, $\Delta_{\mathrm{VDd} / 3 \mathrm{DRD}}$ ranges were smaller than the ones found for $\Delta_{\mathrm{VD} / 3 \mathrm{DRD}}:-1 \%$ to $4 \%$ for case $1,-0.5 \%$ to $1.5 \%$ for case 2 , and $-1.5 \%$ to $2 \%$ for case 3. Furthermore, a linear regression was found between $\Delta_{\mathrm{VDd} / 3 \mathrm{DRD}}$ and $\rho$ only for case 1 , with a smaller slope and correlation coefficient than for $\Delta_{\mathrm{VD} / 3 \mathrm{DRD}}\left(\Delta_{\mathrm{VDd} / 3 \mathrm{DRD}}(\%)=40.5\right.$ $\rho-38.0, R^{2}=0.88$ ) (Fig. $2 \mathrm{~B}$ ), whereas no linear relationship was found for the other cases $\left(R^{2}<0.13\right)$.
The DVHs are presented for the $\mathrm{D}_{3 \mathrm{DRD}}, \mathrm{D}_{\mathrm{VD}}$, and $\mathrm{D}_{\mathrm{VDd}}$ calculations in Figure 3 (case 1) and Figure 4 for the liver tumor and normal liver of case 3 . For case 2, no DVHs were computed because no tumor was present. The DVHs confirm the previous results with close curves for the three $3 \mathrm{D}$ dosimetry calculations in case 3 ( ${ }^{90}$ Y-microspheres). For case 1 ( ${ }^{131}$ I-tositumomab), the density correction did not improve the agreement of the DVHs.

\section{DISCUSSION}

The purpose of this study was to evaluate the accuracy of 3D abdominal dosimetry, assuming the hypothesis of homogeneous tissue density when using a DK approach. A simple density correction at the voxel level was also proposed and evaluated. Our results show a small influence of TDH in the abdominal region for the 3 representative clinical cases studied. Nevertheless, the proposed density correction method improved absorbed dose calculated with DK.

We chose to focus on the abdomen because of the small differences in tissue density and because numerous administrations of TRT are of interest due either to the presence of tumors or to the potential for normal-organ toxicity. Three representative clinical cases were considered. Case 1 was a NHL patient treated with ${ }^{131}$ I-tositumomab. Case 2 was a patient with a clinically suspected NET treated with ${ }^{177} \mathrm{Lu}$-peptide. Case 3 was an $\mathrm{HCC}$ patient treated with ${ }^{90} \mathrm{Y}$-microspheres.

For each case, the absorbed dose calculation was performed with 2 approaches. First, a direct MC method implemented in the software tool 3D-RD (32), taking into account voxel density, was considered as the reference. Second, we used DK, with a revision of the VSV method

TABLE 2

Average Absorbed Dose Comparison for Case 2

\begin{tabular}{|c|c|c|c|c|c|c|c|c|c|c|}
\hline \multirow[b]{3}{*}{ Structure of interest } & \multirow[b]{3}{*}{ TIA (MBq.s) } & \multirow[b]{3}{*}{ Mass $(g$, with $\mathrm{d}(x, y, z))$} & \multirow[b]{3}{*}{ Mass $(\mathrm{g}$, with $\mathrm{d}=1.04)$} & \multicolumn{7}{|c|}{ Average absorbed dose (Gy) and RD (\%) } \\
\hline & & & & \multirow[b]{2}{*}{ 3D-RD } & \multicolumn{2}{|c|}{ VD } & \multicolumn{2}{|c|}{ VDd } & \multicolumn{2}{|c|}{ VDd } \\
\hline & & & & & Gy & $\%$ & Gy & $\%$ & Gy & $\%$ \\
\hline Liver & 621 & 2,220 & 2,251 & 7.05 & 6.90 & -2.2 & 6.98 & -1.1 & 6.99 & -0.9 \\
\hline Spleen & 437 & 318 & 325 & 33.5 & 32.4 & -3.3 & 33.3 & -0.7 & 33.2 & -1.1 \\
\hline Renal cortex & 585 & 503 & 528 & 28.4 & 26.8 & -5.6 & 28.1 & -1.0 & 28.1 & -1.0 \\
\hline Renal medulla & 60.7 & 129 & 133 & 11.9 & 11.5 & -3.3 & 11.9 & 0.1 & 11.9 & 0.1 \\
\hline
\end{tabular}




\begin{tabular}{|c|c|c|c|c|c|c|c|c|c|c|}
\hline \multirow[b]{3}{*}{ Structure of interest } & \multirow[b]{3}{*}{ TIA (MBq.s) } & \multirow[b]{3}{*}{ Mass $(\mathrm{g}$, with $\mathrm{d}(x, y, z))$} & \multirow[b]{3}{*}{ Mass (g, with $\mathrm{d}=1.04)$} & \multicolumn{7}{|c|}{ Average absorbed dose (Gy) and RD (\%) } \\
\hline & & & & \multirow[b]{2}{*}{ 3D-RD } & \multicolumn{2}{|c|}{ VD } & \multicolumn{2}{|c|}{ VDd } & \multicolumn{2}{|c|}{ VDd̄ } \\
\hline & & & & & Gy & $\%$ & Gy & $\%$ & Gy & $\%$ \\
\hline Tumoral liver & 196 & 749 & 743 & 38.2 & 38.8 & 1.7 & 38.5 & 0.8 & 38.5 & 0.8 \\
\hline Nontumoral liver & 86.1 & 1,970 & 1,954 & 6.55 & 6.7 & 1.6 & 6.60 & 0.8 & 6.60 & 0.8 \\
\hline Stomach wall & 1.29 & 226 & 230 & 0.86 & 0.85 & -1.5 & 0.86 & -0.1 & 0.86 & 0.1 \\
\hline
\end{tabular}

(17). This method was implemented in the software tool VoxelDose (or VD) (18), assuming a homogeneous density distribution $\left(\rho=1.04 \mathrm{~g} \cdot \mathrm{cm}^{-3}\right)$. Both implementations (3D$\mathrm{RD}$ and VD) were previously compared in a homogeneous

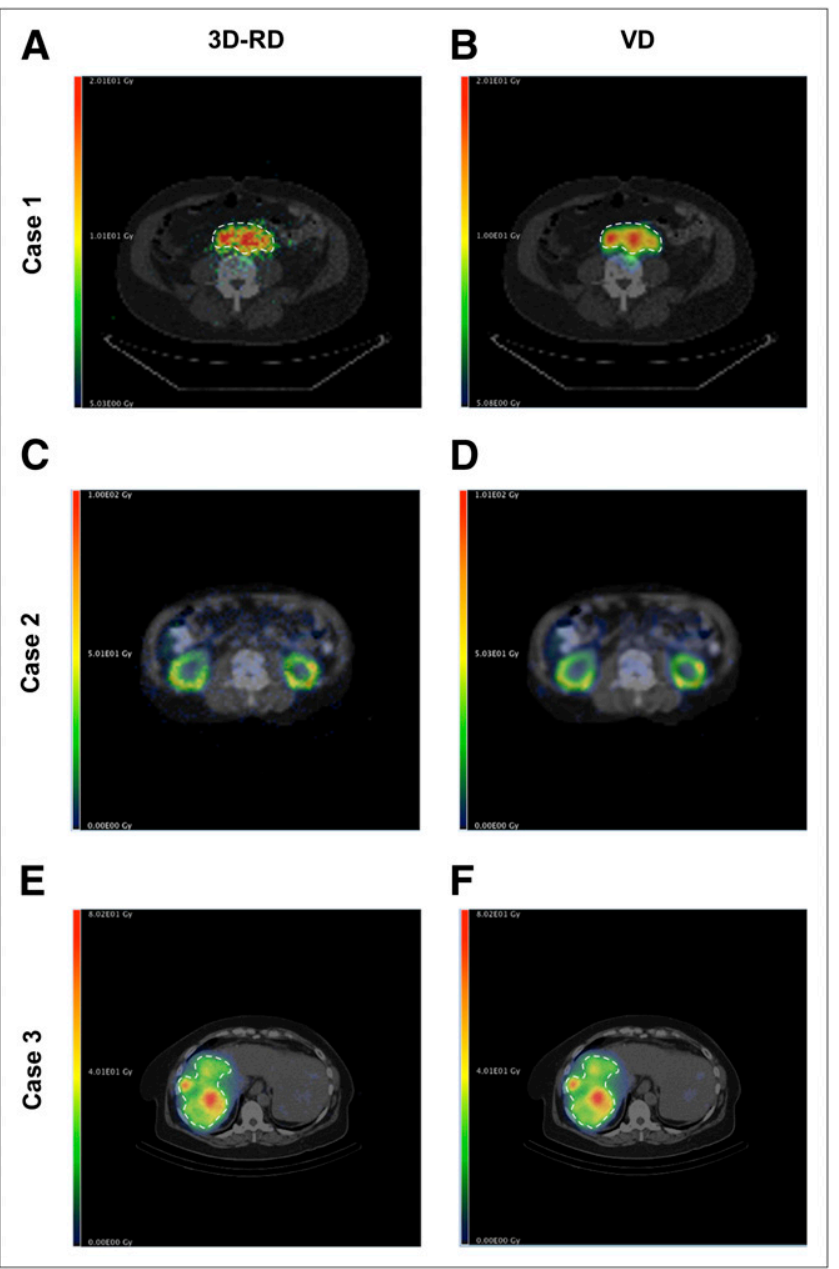

FIGURE 1. Case 1: images of NHL treated with ${ }^{131}$-tositumomab. Transverse slice of absorbed dose calculated with 3D-RD (A) and VoxelDose (B) without density correction (VD) is shown. Main differences between both absorbed dose maps are due to $M C$ statistical fluctuations (A). Case 2: simulation of ${ }^{177}$ Lu-peptide. Transverse slice of absorbed dose calculated with 3D-RD (C) and VoxelDose (D) without density correction (VD) is shown. Case 3: HCC treated with ${ }^{90} \mathrm{Y}$-microspheres. Transverse slice of absorbed dose calculated with 3D-RD $(E)$ and VoxelDose $(F)$ without density correction (VD) is shown. Dashed lines represent tumor boundaries. soft-tissue medium for ${ }^{90} \mathrm{Y}$ and ${ }^{131} \mathrm{I}$, with discrepancies below $1 \%$ (11). Regarding the statistical equivalence of both approaches, the MC simulations were done with $10^{7}$ histories for each decay component, and the VSV kernels were calculated with $10^{9}$ histories (11). Therefore, the VD calculation is statistically equivalent to an $\mathrm{MC}$ simulation with $10^{9}$ histories per voxel. Although the statistical uncertainties between 3D-RD and VD are not equivalent, the voxel-based comparison is not affected, because the absorbed dose differences were binned (toward density) before being plotted against density.

The calculation time with MC was much longer than when using our VD method, but this should not hide the fact that VSVs require long prior calculations and an algorithm to be adapted to the voxel size of the $\gamma$-camera. Furthermore, with DK in general, and VSV in our case, it is not possible to integrate the effect of TDH on the energy deposition. Therefore, a postcalculation density correction is proposed and investigated. Its principle is to correct the absorbed dose for each voxel by scaling it with the real density values derived from CT data. This correction would not adjust the fluency perturbations of primary particles (photons or electrons) but only the local energy deposition of secondary charged particles (electrons).

The organ and tumor level comparison between 3D-RD and VD shows a limited impact of tissue heterogeneities, with relative differences on the average absorbed dose, $\Delta_{\mathrm{VD} / 3 \mathrm{DRD}}$, ranging from $-5.9 \%$ to $1.7 \%$ for the 3 studied clinical cases (Table 1). The results are in agreement with previous results obtained by Buffa et al. (23) and confirm our assumptions.

Slices of the 3D absorbed dose maps computed with 3DRD and VD show similar aspects (Fig. 1). Because of the statistical nature of MC simulations (3D-RD), the corresponding absorbed dose maps are less smoothed than the ones obtained with the VD method.

At the voxel level, the comparison between 3D-RD and VD showed a $\Delta_{\mathrm{VD} / 3 \mathrm{DRD}}$ range of $0 \%$ to $14 \%$. Furthermore, a linear relationship between $\Delta_{\mathrm{VD} / 3 \mathrm{DRD}}$ and the density $\rho$ was found for the 3 clinical cases studied, with $R^{2}$ values over 0.90 and slopes with absolute values higher than 50 ( -91.2 to -55.6$)$. This finding indicated a dependence of $\Delta_{\mathrm{VD} / 3 \mathrm{DRD}}$ on tissue density at the voxel level, although the impact of this dependence and tissue heterogeneities on abdominal dosimetry is likely to be small given the relatively 


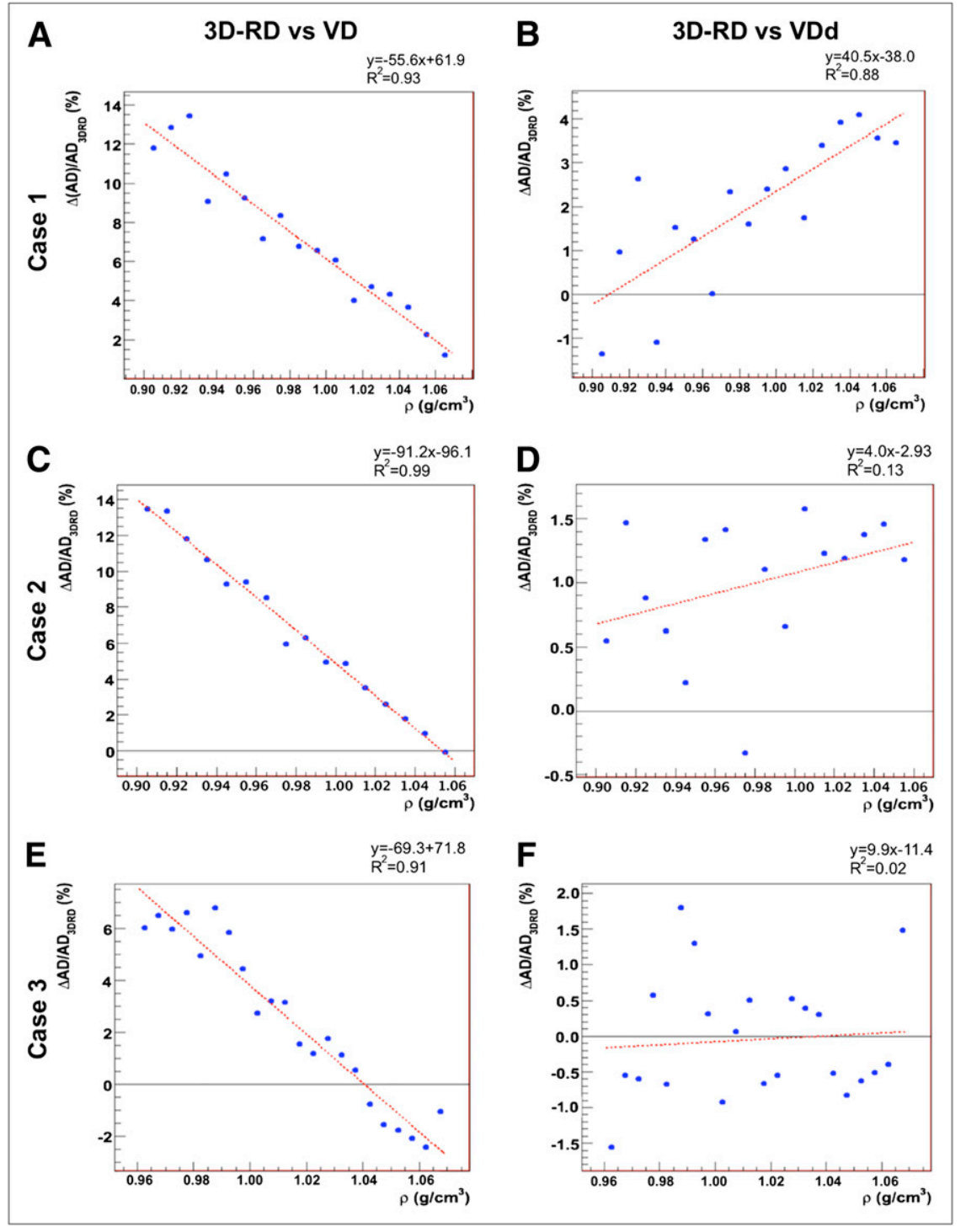

FIGURE 2. Case 1: NHL treated with ${ }^{131} \mid-$ tositumomab. Linear correlation between density-binned average absorbed dose differences $\Delta D$ and density $\rho$, for absorbed doses above 1 Gy and $\rho \geq 0.9 \mathrm{~g} \cdot \mathrm{cm}^{-3}$, is shown. (A) $\Delta \mathrm{D}$ between $3 \mathrm{D}-\mathrm{RD}$ and VoxelDose with homogeneous density (VD). (B) $\Delta \mathrm{D}$ between 3D-RD and VoxelDose with density correction (VDd). Case 2: simulation of ${ }^{177}$ Lu-peptide. Linear correlation between density-binned $\Delta \mathrm{D}$ (average absorbed dose differences) and $\rho$ (density), for absorbed doses above 1 Gy and $\rho \geq 0.9 \mathrm{~g} \cdot \mathrm{cm}^{-3}$, is shown. (C) $\Delta \mathrm{D}$ between $3 \mathrm{D}-\mathrm{RD}$ and VD.(D) $\Delta \mathrm{D}$ between 3D-RD and VDd. Case 3:HCC treated with ${ }^{90} \mathrm{Y}$-microspheres. Linear correlation between density-binned $\Delta \mathrm{D}$ (average absorbed dose differences) and $\rho$ (density), for absorbed doses above 1 Gy and $\rho \geq$ $0.96 \mathrm{~g} \cdot \mathrm{cm}^{-3}$, is shown. (E) $\Delta \mathrm{D}$ between 3D-RD and VD. (F) $\Delta \mathrm{D}$ between 3D-RD and VDd. uniform distribution within the abdomen. DVHs confirm this notion because they show that the density distributions are heavily weighted toward a few average-density bin values;

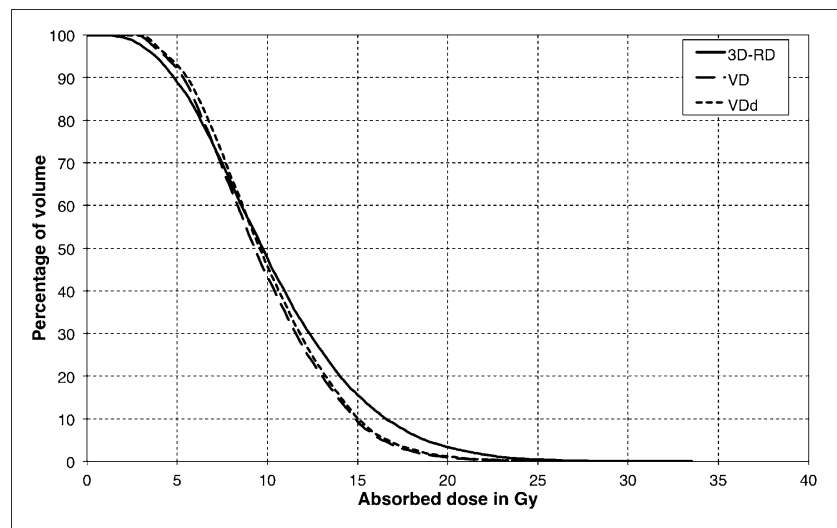

FIGURE 3. Case 1: NHL treated with ${ }^{131}$-tositumomab. DVH in tumor calculated by 3D-RD, VoxelDose with homogeneous density distribution (VD), and with density correction (VDd). these histograms are given as supplemental Figures 1-3 (supplemental materials are available online only at http:// jnm.snmjournals.org).

The density corrections at the voxel and organ levels improved the dose calculation with VD, with $\Delta_{\mathrm{VDd} / 3 \mathrm{DRD}}$ ranging from $-3.1 \%$ to $0.8 \%$ and $\Delta_{\mathrm{VDd} / 3 \mathrm{DRD}}$ ranging from $-3.6 \%$ to $0.8 \%$. The major improvement concerns case 2 , with $\Delta_{\mathrm{VD} / 3 \mathrm{DRD}}$ initially ranging from -5.6 to $-2.2 \%$ and $\Delta_{\mathrm{VDd} / 3 \mathrm{DRD}}$ ranging from $-1.1 \%$ to $0.1 \%$ after correction. In contrast, the density correction for case 3 is of limited interest; because of low relative discrepancies without any correction $\left(\Delta_{\mathrm{VD} / 3 \mathrm{DRD}}\right.$ ranging from $-1.5 \%$ to $\left.1.7 \%\right)$, the relative differences with density correction $\Delta_{\mathrm{VDd} / 3 \mathrm{DRD}}$ were below $1 \%$, which is on the order of the statistical uncertainty of the 3D-RD MC simulation values used as a comparison. Although the MC statistical uncertainties on individual voxel absorbed doses are relatively high (root-mean-square values for the voxel uncertainties are $6.5 \%, 4.7 \%$, and $5.0 \%$ for the 3 cases), the uncertainty on the binned values are 


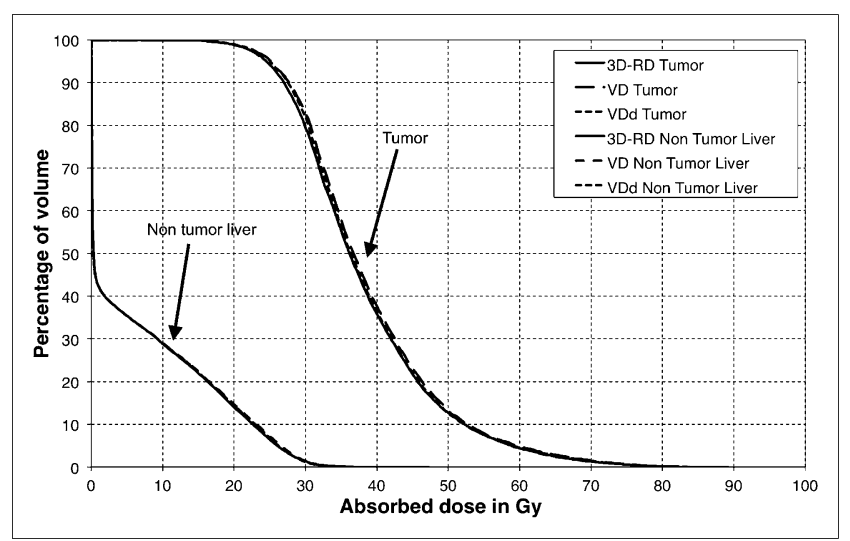

FIGURE 4. Case 3: HCC treated with ${ }^{90}$ Y-microspheres. DVH in and tumoral and nontumoral liver calculated by 3D-RD, VoxelDose with homogeneous density distribution (VD), and with density correction (VDd).

much lower (the maximum bin uncertainties due only to MC statistics are $0.7 \%, 0.4 \%$, and $0.5 \%$ ).

The density correction at the voxel level improved the agreement between 3D-RD and VD at the voxel level. Indeed, the voxel absorbed dose relative difference $\Delta_{\mathrm{VDd} / 3 \mathrm{DRD}}$ range was reduced to $-1.5 \%$ to $4 \%$. Furthermore, no relationship was found between the relative difference $\Delta_{\mathrm{VDd} / 3 \mathrm{DRD}}$ and $\rho$ for ${ }^{177} \mathrm{Lu}$-peptide and ${ }^{90} \mathrm{Y}$-microsphere cases (Figs. 2D and 2F), whereas a linear relationship still existed between $\Delta_{\mathrm{VDd} / 3 \mathrm{DRD}}$ and $\rho$ for the ${ }^{131} \mathrm{I}$-tositumomab case but with a smaller slope than with $\Delta_{\mathrm{VD} / 3 \mathrm{DRD}}(40.5$ vs. -55.6 , respectively; Figs. 2A and 2B) and a smaller correlation coefficient ( 0.88 vs. 0.93 , respectively). This result can be explained by the fact that the proposed density correction is more effective for nonpenetrating particles and thus for preponderant $\beta^{-}$emissions $\left({ }^{90} \mathrm{Y}\right.$ and $\left.{ }^{177} \mathrm{Lu}\right)$. When looking at Figures 2A, 2C, and 2E, the difference between VD and MC for $\rho=1.04 \mathrm{~g} \cdot \mathrm{cm}^{-3}$ is near zero for case $3,1 \%$ for case 2 , and $4 \%$ for case 1 , meaning that the influence of tissue heterogeneities does not depend only on the local energy deposition for these 2 latter cases. Indeed, the yield of the photon component is greater for ${ }^{131} \mathrm{I}$ (yield, 1.0) than ${ }^{177} \mathrm{Lu}$ (yield, 0.17) and null for ${ }^{90} \mathrm{Y}$. For a comprehensive density correction, the cross-dose component would have to be treated separately as it is more affected by density heterogeneities at a larger scale (tissue, suborgan).

Our results are valid in the conditions we met-that is, the abdominal region and radionuclides with major dose contribution coming from the electron emissions (atomic and $\beta^{-}$). The comparison was done with specific MC and DK implementations, namely 3D-RD and VoxelDose, respectively. 3D-RD was chosen as the gold standard with the advantage of being well established, with a history of clinically relevant publications.

Further studies will have to be undertaken with different conditions for any extension of these results to other treatment localizations, radionuclides. Additionally, the implementation of scaling methods that were proposed for internal dosimetry (25-27) could be investigated in other conditions - that is, with a major photon emission component or with sites in the thoracic region or bone.

\section{CONCLUSION}

There is a small but significant influence of tissue density heterogeneity on the absorbed dose calculation in the abdominal region for radionuclides with a preponderant $\beta$-emission (i.e., ${ }^{131} \mathrm{I},{ }^{177} \mathrm{Lu}$, and ${ }^{90} \mathrm{Y}$ ). A simple density correction at the voxel level was proposed for DK methods, allowing improvement of the absorbed dose calculation.

\section{DISCLOSURE}

The costs of publication of this article were defrayed in part by the payment of page charges. Therefore, and solely to indicate this fact, this article is hereby marked "advertisement" in accordance with 18 USC section 1734. No potential conflict of interest relevant to this article was reported.

\section{REFERENCES}

1. Robbins RJ, Schlumberger MJ. The evolving role of ${ }^{131} \mathrm{I}$ for the treatment of differentiated thyroid carcinoma. $J$ Nucl Med. 2005;46(suppl 1):28S-37S.

2. Wahl RL. Tositumomab and ${ }^{131} \mathrm{I}$ therapy in non-Hodgkin's lymphoma. $\mathrm{J} \mathrm{Nucl}$ Med. 2005;46(suppl 1):128S-140S.

3. Flux G, Bardies M, Chiesa C, et al. Clinical radionuclide therapy dosimetry: the quest for the "Holy Gray". Eur J Nucl Med Mol Imaging. 2007;34:1699-1700.

4. Brans B, Bodei L, Giammarile F, et al. Clinical radionuclide therapy dosimetry: the quest for the "Holy Gray.". Eur J Nucl Med Mol Imaging. 2007;34:772786.

5. Loevinger R, Budinger TF, Watson EE. MIRD Primer For Absorbed Dose Calculations. Revised. New York, NY: The Society of Nuclear Medicine; 1991.

6. Bouchet LG, Bolch WE, Weber DA, Atkins HL, Poston JW. MIRD Pamphlet no. 15: radionuclide $S$ values in a revised dosimetric model of the adult head and brain. Medical Internal Radiation Dose. J Nucl Med. 1999;40:62S-101S.

7. Bouchet LG, Bolch WE, Blanco HP, et al. MIRD pamphlet no 19: absorbed fractions and radionuclide $\mathrm{S}$ values for six age-dependent multiregion models of the kidney. J Nucl Med. 2003;44:1113-1147.

8. Snyder W, Ford M, Warner G. MIRD Pamphlet No. 5: Estimates of Absorbed Fractions for Monoenergetic Photon Sources Uniformly Distributed in Various Organs of a Heterogeneous Phantom. Revised. New York, NY: The Society of Nuclear Medicine; 1978.

9. Flux G, Bardies M, Monsieurs M, et al. The impact of PET and SPECT on dosimetry for targeted radionuclide therapy. Z Med Phys. 2006;16:47-59.

10. G Stabin MG, Sparks RB, Crowe E. OLINDA/EXM: the second-generation personal computer software for internal dose assessment in nuclear medicine. J Nucl Med. 2005;46:1023-1027.

11. Dieudonné A, Hobbs RF, Bolch WE, Sgouros G, Gardin I. Fine-resolution voxel $\mathrm{S}$ values for constructing absorbed dose distributions at variable voxel size. J Nucl Med. 2010;51:1600-1607.

12. Divoli A, Chiavassa S, Ferrer L, Barbet J, Flux GD, Bardies M. Effect of patient morphology on dosimetric calculations for internal irradiation as assessed by comparisons of Monte Carlo versus conventional methodologies. J Nucl Med. 2009;50:316-323.

13. Prideaux AR, Song H, Hobbs RF, et al. Three-dimensional radiobiologic dosimetry: application of radiobiologic modeling to patient-specific 3-dimensional imaging-based internal dosimetry. J Nucl Med. 2007;48:1008-1016.

14. Ljungberg M, Sjögreen Gleisner K, Liu X, Frey EC, Dewaraja YK, Strand S-E. A 3-dimensional absorbed dose calculation method based on quantitative SPECT for radionuclide therapy: evaluation for ${ }^{131} \mathrm{I}$ using Monte Carlo simulation. J Nucl Med. 2002;43:1101-1109.

15. Chiavassa S, Bardies M, Guiraud-Vitaux F, et al. OEDIPE: a personalized dosimetric tool associating voxel-based models with MCNPX. Cancer Biother Radiopharm. 2005;20:325-332.

16. Berger MJ. Distribution of absorbed dose around point sources of electrons and beta particles in water and other media. J Nucl Med. 1971;12:5-23. 
17. Bolch WE, Bouchet LG, Robertson JS, et al. MIRD pamphlet no. 17: the dosimetry of nonuniform activity distributions-radionuclide $\mathrm{S}$ values at the voxel level. Medical Internal Radiation Dose Committee. J Nucl Med. 1999;40:11S-36S.

18. Gardin I, Bouchet LG, Assié K, et al. Voxeldose: a computer program for 3-D dose calculation in therapeutic nuclear medicine. Cancer Biother Radiopharm. 2003;18:109-115.

19. Guy MJ, Flux GD, Papavasileiou P, Flower MA, Ott RJ. RMDP: a dedicated package for ${ }^{131}$ I SPECT quantification, registration and patient-specific dosimetry. Cancer Biother Radiopharm. 2003;18:61-69.

20. Akabani G, Hawkins WG, Eckblade MB, Leichner PK. Patient-specific dosimetry using quantitative SPECT imaging and three-dimensional discrete Fourier transform convolution. J Nucl Med. 1997;38:308-314.

21. Buffa FM, Verhaegen F, Flux GD, Dearnaley DP. A Monte-Carlo method for interface dosimetry of beta emitters. Cancer Biother Radiopharm. 2003;18:463471.

22. Kwok CS, Bialobzyski PJ, Yu SK, Prestwich WV. Effect of tissue inhomogeneity on dose distribution of point sources of low-energy electrons. Med Phys. 1990;17:786-793.

23. Buffa FM, Verhaegen F. Backscatter and dose perturbations for low- to mediumenergy electron point sources at the interface between materials with different atomic numbers. Radiat Res. 2004;162:693-701.

24. Cho SH, Reece WD. Monte Carlo calculations of the dose backscatter factor for monoenergetic electrons. Phys Med Biol. 1999;44:13-26.

25. Janicki C, Duggan DM, Gonzalez A, Coffey CW, Rahdert DA. Dose model for a beta-emitting stent in a realistic artery consisting of soft tissue and plaque. Med Phys. 1999;26:2451-2460.

26. Loudos G, Tsougos I, Boukis S, et al. A radionuclide dosimetry toolkit based on material-specific Monte Carlo dose kernels. Nucl Med Commun. 2009;30:504512.

27. Furhang EE, Chui CS, Sgouros G. A Monte Carlo approach to patient-specific dosimetry. Med Phys. 1996;23:1523-1529.

28. Wiseman GA, Kornmehl E, Leigh B, et al. Radiation dosimetry results and safety correlations from ${ }^{90} \mathrm{Y}$-ibritumomab tiuxetan radioimmunotherapy for relapsed or refractory non-Hodgkin's lymphoma: combined data from 4 clinical trials. $J$ Nucl Med. 2003;44:465-474.

29. Kwekkeboom DJ, Mueller-Brand J, Paganelli G, et al. Overview of results of peptide receptor radionuclide therapy with 3 radiolabeled somatostatin analogs. J Nucl Med. 2005;46(suppl 1):62S-66S.

30. Salem R, Thurston KG. Radioembolization with Yttrium 90 microspheres: a state-of-the-art brachytherapy treatment for primary and secondary liver malignancies. Part 1: Technical and methodologic considerations. J Vasc Interv Radiol. 2006;17:1251-1278.

31. Eckerman KF, Endo A. MIRD: Radionuclide Data and Decay Schemes. Reston, VA: Society of Nuclear Medicine; 2007.

32. Hobbs RF, Wahl RL, Lodge MA, et al. ${ }^{124}$ I PET-based 3D-RD dosimetry for a pediatric thyroid cancer patient: real-time treatment planning and methodologic comparison. J Nucl Med. 2009;50:1844-1847.

33. Hendricks JS, McKinney GW, Waters SW. MCNPX Extended Version 2.5.0. Manual. Los Alamos, NM: Los Alamos National Laboratory; 2005.

34. International Commission on Radiation Units and Measurements (ICRU). Photon, Electron, Proton and Neutron Interaction Data for Body Tissues. Report 46. Techreport. Bethesda, MD: ICRU; 1992.

35. Hobbs RF, Baechler S, Wahl RL, et al. Arterial wall dosimetry for non-Hodgkin lymphoma patients treated with radioimmunotherapy. J Nucl Med. 2010;51:368375 .

36. He B, Du Y, Song X, Segars WP, Frey EC. A Monte Carlo and physical phantom evaluation of quantitative In-111 SPECT. Phys Med Biol. 2005;50:4169-4185.

37. He B, Frey EC. Comparison of conventional, model-based quantitative planar, and quantitative SPECT image processing methods for organ activity estimation using In-111 agents. Phys Med Biol. 2006;51:3967-3981.

38. Baechler S, Hobbs R, Bischof-Delaloye A, et al. The use of 3D patient-specific radiobiological dosimetry for kidneys in peptide receptor radionuclide therapy [abstract]. J Nucl Med. 2009;50(suppl 2):498.

39. Dieudonné A, Garin E, Laffont S, et al. Clinical feasibility of fast 3-dimensional dosimetry of the liver for treatment planning of hepatocellular carcinoma with ${ }^{90}$ Y-microspheres. J Nucl Med. 2011;52:1930-1937. 